\title{
The Study of m6A, m5C, m1A, m7G, Adenosine-to-Inosine and Pseudouridine RNA Modification-related Molecules in Peripheral Blood of Rheumatoid Arthritis
}

\author{
Ziqing Yu \\ First Affiliated Hospital of Fujian Medical University \\ Jifeng Tang \\ First Affiliated Hospital of Fujian Medical University \\ Jinfang Xia \\ First Affiliated Hospital of Fujian Medical University \\ Yinfeng Xiao \\ First Affiliated Hospital of Fujian Medical University \\ Yujue He \\ First Affiliated Hospital of Fujian Medical University \\ Renquan Jiang \\ First Affiliated Hospital of Fujian Medical University \\ Shuhui Chen \\ First Affiliated Hospital of Fujian Medical University \\ Jinpiao Lin ( $\square$ jinpiaolin@fjmu.edu.cn ) \\ First Affiliated Hospital of Fujian Medical University
}

Research article

Keywords: Rheumatoid arthritis, m6A, m5C, m1A, m7G, I, $\psi$

Posted Date: October 26th, 2021

DOI: https://doi.org/10.21203/rs.3.rs-964198/v1

License: (c) (i) This work is licensed under a Creative Commons Attribution 4.0 International License. Read Full License 


\section{Abstract Background}

The overall situation of RNA epigenetics in rheumatoid arthritis (RA) is still unclear. This study aims to investigate the expression level of m6A, m5C, m1A, m7G, adenosine-to-inosine, pseudouridine $(\psi)$ modification-related molecules in peripheral blood of RA.

\section{Methods}

33 RA patients and 36 healthy donors (HD) were included in this study. Gene expression levels were detected by real time-polymerase chain reaction (RT-PCR). Correlation analyses were performed by Pearson correlation test. Least absolute shrinkage and selection operator regression (LASSO) regression and Logistic regression were used for seeking risk factors.

\section{Results}

The expression levels of METTL3, TET2, ADAR1 increased while ALKBH5, TRMT10C TRMT61B ALKBH3 ALYREF METTL 1 decreased in RA patients. Analysis shown 47 weak correlations, 43 moderate correlations and 2 strong correlations existed among these molecules. Area under curve (AUC) of ALYREF and TET2 combined ROC was 0.976 which indicated them were the most valuable risk factors in RA. The expression of ALKBH3 and TRMT10C was higher in ESR-negative RA patients, while the expression of METTL 1 and METTL3 was higher in RF-negative group or CRP-negative group respectively. Besides, the level of METTL3 and TRMT10C was increased in DAS-28-ESR. Furthermore, METTL3 level was negative related with the levels of RF and DAS28-ESR. The level of TRMT10C was negative with ESR and DAS-28-ESR levels, and $A L K B H 5$ expression was positive related with CRP level. The differential expression genes were mostly correlated with PLT and MCHC of blood routine examination.

\section{Conclusions}

Our study constructed a network of six kinds of mRNA modifications in RA. We found multiple mRNA-modification-related genes were different expression between RA patients and HD. Complicated mutual relations existed among these genes. ALYREF and TET2 were the most valuable risk factors in RA. This work hopes to support a new perspective for clarifying pathogenic mechanism of RA.

\section{Background}

Rheumatoid arthritis (RA) is a chronic inflammatory autoimmune disease. Large numbers of inflammatory cells infiltration and excessive proliferation in synovial tissue are the main characteristics of RA, which damage the joint bone and cartilage and follow by causing progressive destruction of the joint. The accumulating joint injuries will lead to joint deformity, even irreversible disability. Due to the complex influencing factors and genetically susceptibility, the exact etiologies and pathogenesis of RA are still unclear[1].

Epitranscriptomic, also known as "RNA epigenetics", is an important mechanism of epigenetic regulation. RNA modification is a dynamic and reversible process which is regulated by "writers", "erasers" and effector proteins "readers". In the advance of high-through sequencing detection technologies, the functional study of epitranscriptomic has been greatly precipitated, which supports a new layer to progression of various diseases[2]. Increased studies have demonstrated that kinds of RNA modifications play important roles in occurrence and development of multiple diseases. But the situations of epitranscriptomic in RA haven't been investigated exactly. Even though more than 170 types of chemical modifications have been discovered in RNA until now, there are only handful of modifications are detected in mRNA[3]. Based on current reports, our work contains a majority of mRNA-related modification, including N6-methyladenosine (m6A), N1methyladenosine (m1A), 5-methylcytosine (m5C), N7-methylguanosine (m7G), pseudouridine $(\Psi)$ and adenosine-to-inosine (A-to-I) [4-8]. There are numbers of studies demonstrated these modifications push forward an immense influence on mRNA metabolism and have closed connection with many kinds of diseases.

The famous and crucial modifications occur on adenine, contain m6A, m1A, A-to-I and so on. m6A is methylation at the sixth nitrogen atom of RNA base $A$. It is the most abundant and common post transcription modification in eukaryotic mRNA. No less than 1-2 methylated adenosines in every single mRNA. It is catalyzed by a methyltransferase complex that consists of METTL3, WTAP and other subunits while demethylated by "erasers" FTO and ALKBH5. Many lines of evidence indicate that m6A exerts an enormous function on mRNA splicing, export, stability, translation[9]. m1A is analogous to m6A, with the methyl attaches to the N1[7]. The methyltransferase consists with TRMT6TRMT61A which is responsible for the installation of m1A in mRNA[10], while the other complex, TRMT61B-TRMT10C[11], catalyzes the m1A 
in mitochondrial mRNA. The reversal of $\mathrm{m} 1 \mathrm{~A}$ in RNA can be catalyzed by ALKBH1 and ALKBH3[12]. Although the protein that recognize m1A haven't been well studied, researchers have proved m1A can promote the start of translation. The common type of RNA editing is A-to-I, which is catalyzed by ADAR family enzymes, including ADAR1, ADAR2 and so on. Primary, ADAR enzymes affect the structure of dsRNA, which formed by pre-mRNA [13]. Besides, the A-to-I editing can change the amino acids and affect other transcription processes[14].

$\Psi$ is the most abundance modifications in total RNA and widely presents in mRNA. Like A-to-I modification, $\Psi$ modification can alter the codons. The formation of $\Psi$ is main catalyzed by pseudouridinesynthases (PUSs). In human, PUS1, PUS7, TRUB1, TRUB2 have been reported to catalyze a subset of $\Psi$ in mRNA[15-17].

$\mathrm{m7G}$, the most recognized manners in mRNA modifications, plays an integral role in mRNA metabolism. It presents in not only the cap of mRNA, but also be found in the sequence coding for amino acids in protein (CDS) of mRNA[18]. The METTL1-WDR4 [19]complex and RNMT[20] are the main "writers". Knowledge is limited about the protein responsible for removing or recognizing m7G.

$\mathrm{m} 5 \mathrm{C}$ is one of the most widely known post-transcriptional modifications. It exists in both DNA and RNA in mammal. Previous studies have shown the importance of $\mathrm{m} 5 \mathrm{C}$ modification in DNA. However, with the development of technologies, m5C modification on RNA has been more and more discussed. NSUN2 and DNMT2 are the main m5C "writers", which are main applied on mRNA and tRNA respectively. m5C can be oxidized by TET family enzymes, mainly by TET2. In mRNA, YBX1 and ALYREF are the primary "readers" of m5C. Like m6A, m5C affects the expression of proteins by regulating mRNA metabolism, including both translation efficiency and transcript export[21].

A number of researches manifest RNA modifications make effect on the function of immune system. For instance, METTL3-mediated m6A level was associated with the activation of dendritic cells, macrophage and was vital for maintaining T cell homeostasis and differentiation[22-24]. The level of $\mathrm{m} 5 \mathrm{C}$ in IL-17 mRNA could promote the translation to increase the expression of IL-17[25], and changed m5C level of mRNA was associated with disease activated in systemic lupus erythematosus (SLE)[26]. m7G in mRNA could active Type I IFN response[27]. ADAR1-mediated modification was related with cytosolic innate immune[13]. All of these evidences indicated RNA modifications might exert a function on the development and progression of autoimmune diseases. Our work mainly detected the expression of these modifications and discussed the relationships with clinical variables in RA in order to furnish a new sight of understanding the pathogenetic mechanisms of RA.

\section{Methods}

\section{Study Population}

Thirty-three RA patients and thirty-six healthy donors were enrolled at the First Affiliated Hospital of Fujian Medical University from January 2021 to February 2021. Each RA patient recruited for this study satisfied the 2010 American College of Rheumatology/European League against Rheumatism criteria. In addition, 36 healthy donors without a clinical diagnosis of any inflammatory relation to patients of autoimmune disease were admitted to this study. All participants who had history of cardiovascular disease, endocrine disease, hepatic disease, renal disease and any other chronic inflammatory diseases were excluded. The study had from the Ethics Committee of the First Affiliated Hospital of Fujian Medical University. Full informed consents for data collection were obtained from all participants. Characteristics of all patients and healthy donors are presented in detail in Table 1. 
Table 1

Clinical characteristics of volunteers with rheumatoid arthritis (RA) and healthy donors (HD).

\begin{tabular}{|lll|}
\hline Characteristics & RA & HD \\
\hline Number & 33 & 36 \\
\hline Age (years) & $52.09 \pm 12.11$ & $50.53 \pm 8.14$ \\
\hline Sex $(\mathrm{M} / \mathrm{F})$ & $5 / 28$ & $17 / 19$ \\
\hline Anti-CCP(RU/ml) & $129.80(85.55,147.55)$ & $/$ \\
\hline CRP $(\mathrm{mg} / \mathrm{L})$ & $3.88(3.82,11.78)$ & $/$ \\
\hline RF(IU/ml) & $55.7(23.05,363)$ & $/$ \\
\hline ESR $(\mathrm{mm} / \mathrm{h})$ & $33(16,47)$ & $/$ \\
\hline DAS28-ESR & $3.89 \pm 0.99$ & $/$
\end{tabular}

RA: rheumatoid arthritis, HD: healthy donors, CRP: C-reactive protein, ESR: erythrocyte sedimentation rate, Anti-CCP: anti-cyclic citrullinated peptide antibody, RF: rheumatoid factor, DAS28: disease Activity Score 28.

All values are represented as the mean \pm standard deviation or the median (interquartile range) depending on whether the parameters follow a normal distribution.

\section{Total Rna Extraction And Real-time Pcr Analysis}

Peripheral blood was collected in EDTA-anticoagulated vacutainer tubes. Red Blood Cell Lysing buffer (BD Biosciences, USA) was used for red cell lysis. Total RNAs were extracted by TRIzol (Ambion, USA). Complementary DNA (cDNA) was synthesized using the Thermo Scientific Revert Aid First Strand cDNA Synthesis Kit (Thermo Scientific). The qRT-PCR was done with SybrGreen PCR Master Mix (Vazyme, China) and Quantstudio $^{\text {TM }}$ DX Real-Time PCR Instrument (Applied Biosystems Inc, USA). All primers sequences mentioned above are listed in Supplementary TABLE S1. Data were analyzed with QuantStudio Real-Time PCR software (Applied Biosystems Inc.). The relative levels of transcripts were derived by the $2^{-\Delta \Delta C t}$ method.

\section{Analyses Of Clinical Parameters}

The items in blood routine were tested using a Siemens ADVIA 2120i analyser (Siemens Healthcare Diagnostics, Germany). Erythrocyte sedimentation rate (ESR) was determined using Westergren's method. Serum anti-CCP level was measured using a commercial ELISA kit (EUROIMMUN, Germany) according to the manufacturer's instruction. The levels of rheumatoid factor (RF) and C-reactive protein (CRP) were detected by immunoturbidimetric assay (Dade Behring, Germany). The 28-joint disease activity score (DAS-28) based on ESR was calculated according to the recommendations from the European League Against Rheumatism (EULAR) for patients. The composite Scores DAS28-ESR include the numbers of tender and swollen joints out of 28 joints, the patient's estimation of his/her general health on a visual analog scale (VAS-GH), and either ESR. DAS28-ESR was calculated as $0.56 \times \sqrt{ }($ Tender joint count $)+0.28 \times \sqrt{ }$ (Swollen joint count $)+0.7 \times \ln$ ESR $+0.014 \times$ VAS-GH.

\section{Statistical Analyses}

The analyses in this work were performed in R software version 3.6.3 (R Foundation for Statistical Computing, Austria). All values were represented as the mean \pm standard deviation or the median (interquartile range) depending on whether the parameters follow a normal distribution. Student's t-test or the MannWhitney U-test was used to compare the data according to the normality. Correlation analyses were performed by Pearson correlation test. Figures were constructed with ggplot2 package and ggcorrplot package in R software. For the reason of seeking risk factors for RA, least absolute shrinkage and selection operator regression (LASSO) regression and Logistic regression were used. Glmnet package and pROC package were used respectively in R to conducted LASSO analysis and receiver operating characteristic curve (ROC). $95 \%$ confidence Interval (Cl), odds Ratio (OR) and area under curve (AUC) were provided by R software. A two-tailed $p<0.05$ was deemed to indicate statistical significance.

\section{Results}

Differential Expression Screening of m1A, m6A, m5C, m7G, $\Psi$ and I Related Molecules in Peripheral Blood from RA Patients. 
A total of 25 RNA modification-related protein, including four m6A modification related molecules, six m1A modification related molecules, five $\mathrm{m} 5 \mathrm{C}$ related modification molecules, three $\mathrm{m} 7 \mathrm{G}$ modification related molecules, two A-to-I related molecules and five $\psi$ modification related molecules, were contained in this study. In order to assessed these molecules' expression between RA patients and HD, the mRNA level of these genes in peripheral was determined by RT-PCR. As shown in Figure 1, the expression levels of m6A "writer" METTL3, the m5C "eraser" TET2, the A-to-I "writer" $A D A R 1$ were significantly higher in RA than that in $\mathrm{HD}$, while the expression of m6A "eraser" $A L K B H 5$, the $\mathrm{m} 1 \mathrm{~A}$ "writers" TRMT10C and TRMT61B and "eraser" ALKBH3, the m5C "reader" ALYREF, the m7G modification "writer" METTL 1 were decreased in RA patients. The other clinical characteristics of all participants were summarized in Table 1. Moreover, further analysis demonstrated that the levels of these genes were not associated with age or gender (data not shown).

\section{Correlation among Six Kinds of RNA Modification-related Molecules in RA Patients.}

In order to obtain a general understanding of the expression pattern of different RNA modification-related molecules and explore the relationship among these genes in RA, we calculated pairwise correlations among the expression of 25 genes. We defined that the correlation coefficient greater than 0.8 was strong correlation, the correlation coefficient between 0.5 and 0.8 was moderate correlation, and the correlation coefficient less than 0.5 was weak correlation. Data was shown in Figure 2, there were 47 weak correlations, 43 moderate correlations and 2 strong correlations existed among these molecules. Notably, a strong positive correlation was seen between the levels of $A L Y R E F$ and WTAP $(r=0.9, p<0.05)$. Besides, the expression of FTO presented a strong positive correlation with the expression of TRMT6 ( $r=0.95, p<0.05)$. Not only the expression of RNA modification molecules was remarkably correlated in the same category, but also a significant correlation was present among different types of modification molecules. These results indicated m6A emerged with potential links to m5C and m1 A in RA.

\section{The Expression of ALYREF and TET2 in Peripheral Blood were Protective or Risk Factors Respective for RA.}

With the purpose of identifying risk factors for RA, LASSO regression and Logistic regression were used. Considering the amounts of samples and measured genes and the multicollinearity of data in this work (Fig. 2), we chose LASSO regression analysis to compressed variables first. Twenty-seven variables were involved in LASSO regression analysis, including years, gender and 25 RNA modification-related genes. The result shown only 8 genes were remained in the model, containing METTL3, ALKBH5, WTAP, TRMT10C, ALYREF, TET2, YBX1 and ADAR1 (Figure 3A, 3B). After that, we used Logistic regression analysis to explore the risk factors further. Result demonstrated that the mRNA expression of $T E T 2$ in peripheral blood was risk factor $(\mathrm{OR}>1)$ and the level of $A L Y R E F$ was protective factor for RA (OR<1) (Table 2). Moreover, in order to evaluate the prediction ability of these two factors, ROC analysis was draw. The cut-off value (0.7) was verified by ROC curve. As shown, they achieved very high-performance in distinguishing RA patients and HD (Figure 3C).

Table 2

Logistic regression analysis.

\begin{tabular}{|llll|}
\hline & Odds Ratio & $95 \%$ Cl & P value \\
\hline ALKBH5 & $6.7466 * 10^{-16}$ & $3.3878 * 10^{-32}-13.4354$ & 0.0681 \\
\hline ALYREF & $2.4929 * 10^{-24}$ & $3.1753 * 10^{-42}-0.0000$ & $0.0097 * *$ \\
\hline TET2 & $5.000 * 10^{17}$ & $30419.9510-8.2341 * 10^{30}$ & $0.0087 * *$ \\
\hline The asterisk indicates statistically significant results. ${ }^{*} p<0.05, * * p<0.01$. \\
\hline
\end{tabular}

\section{Correlation of the Expression Levels of Differential Genes with RF, anti-CCP, ESR, CRP and DAS28-ESR.}

In above, we sifted nine differential expression genes, including METTL3, ALKBH5, TET2, ALYREF, TRMT10C, TRMT61B, ALKBH3, METTL1 and $A D A R 1$, between RA and HD. I=In the ACR/EULAR 2010 classification criteria, RF, anti-CCP, ESR and CRP were the main laboratory indicators for evaluating RA progression and disease activity. For the sake of elucidated the relationships between these four clinical parameters and differential expression genes, RA patients were divided into different groups according to the negative and positive of each indicator. The results were shown in Table 3. Compared to the group of RF-negative, the mRNA expression level of METTL 1 was higher in RFpositive group. The mRNA expression level of METTL3 was decreased in CRP-positive group compared with CRP-negative group. Together, the expressions of TRMT10C and $A L K B H 3$ were reduced in ESR-positive group contrast with ESR-negative group. However, there was no difference between anti-CCP-positive group and anti-CCP-negative group among these nine genes. DAS28-ESR is the most widely used scoring system in monitoring RA disease activity. In this study, RA patients were divided into two subgroups with remission-to-low disease activity (DAS28ESR<3.2) and high disease activity (DAS28-ESR $\geq 3.2$ ). As shown in Table 3, we found the expression of METTL3 and TRMT10C were increased in low-disease-activity group compared with high-disease-activity group. 
Further, we used Pearson correlation test to analyze these data. As shown in Table 4, the mRNA expression level of METTL3 was negative correlated with RF $(r=-0.437, p=0.0371)$ and DAS28-ESR $(r=-0.3627, p=0.0380)$, the expression of TRMT10C was negative correlated with ESR $(r=-0.4473, p=0.0091)$ and DAS28-ESR $(r=-0.5332, p=0.0014)$. CRP was positive with ALKBH5 mRNA level $(r=0.4139, p=0.0443)$. Besides, no significant linear correlation was found between other genes and these indicators $(p>0.05)$.

Table 4

Correlation of mRNA expression levels of METTL3, ALKBH5, TET2, ALYREF, TRMT10C, TRMT61B, ALKBH3, METTL1 and ADAR1 with RF, antiCCP, ESR and CRP of RA

\begin{tabular}{|c|c|c|c|c|c|c|c|c|c|c|}
\hline \multirow[t]{2}{*}{ Gene } & \multicolumn{2}{|l|}{ RF } & \multicolumn{2}{|c|}{ Anti-CCP } & \multicolumn{2}{|l|}{ ESR } & \multicolumn{2}{|l|}{ CRP } & \multicolumn{2}{|c|}{ DAS28-ESR } \\
\hline & $r$ & $\mathrm{p}$ & $r$ & $p$ & $r$ & $p$ & $r$ & $\mathbf{p}$ & $\mathbf{r}$ & $\mathbf{p}$ \\
\hline METTL3 & -0.4370 & $0.0371^{*}$ & -0.1198 & 0.5770 & -0.2840 & 0.1092 & -0.1702 & 0.4058 & -0.3627 & $0.0380 *$ \\
\hline ALKBH5 & 0.0030 & 0.9893 & 0.0290 & 0.8929 & 0.1086 & 0.5474 & 0.4139 & $0.0443^{*}$ & 0.1552 & 0.3886 \\
\hline ALKBH3 & -0.3637 & 0.0961 & 0.1182 & 0.5910 & -0.2039 & 0.2630 & 0.0347 & 0.8721 & -0.2167 & 0.2335 \\
\hline ALYREF & -0.1724 & 0.4316 & 0.0423 & 0.8445 & 0.1376 & 0.4452 & 0.0850 & 0.7346 & 0.0870 & 0.6302 \\
\hline TET2 & -0.0858 & 0.6970 & -0.2406 & 0.2573 & 0.0044 & 0.9805 & 0.2897 & 0.1601 & 0.1887 & 0.2928 \\
\hline TRMT10C & -0.3469 & 0.1049 & 0.1930 & 0.3663 & -0.4473 & $0.0091^{\star \star}$ & -0.2997 & 0.1455 & -0.5332 & $0.0014^{* *}$ \\
\hline TRMT61B & -0.2190 & 0.3154 & 0.2947 & 0.1621 & 0.0597 & 0.7413 & 0.0353 & 0.8669 & -0.0337 & 0.8522 \\
\hline ADAR1 & -0.1255 & 0.5683 & 0.0917 & 0.6700 & -0.1436 & 0.4253 & -0.2974 & 0.1583 & 0.0025 & 0.9891 \\
\hline METTL1 & -0.1073 & 0.6260 & 0.0260 & 0.9042 & 0.0856 & 0.6359 & 0.3033 & 0.1497 & -0.0294 & 0.8708 \\
\hline
\end{tabular}

\section{Correlation between Clinical Blood Routine Indicators and Differential Genes in RA Patients.}

Correlation tests had accessed to evaluate the relevance between the clinical blood routine parameters and differential genes. Data was shown in Table 5. The level of METTL3 had had positive correlation with platelet distribution width (PDW) ( $r=0.4307, p=0.0123)$. The level of $A L K B H 5$ was negative related with platelet count (PLT) $(r=-0.4340, p=0.0116)$. and PLT\% $(r=-0.4097, p=0.0179)$, moreover, was positive with Mean Platelet Mass (MPM) $(r=0.3562, p=0.0419)$. The negative association between $A L Y R E F$ level and mean corpusular hemoglobin concerntration $(\mathrm{MCHC})$ was be observed $(r=-0.3663, p=0.0361)$. The negative correlation existed between TRMT61B and MCHC $(r=-0.3524$, $p=0.0443)$.

\section{Discussion}

With the development of detection technology, RNA epitranscriptomics is discovered playing an important role in metabolism of mRNA. RA is an autoimmune disease with unknown pathogenesis. Patients with RA have a complex array of abnormalities involving their immune system. Recent researches hint RNA modification participated in the procession of autoimmune diseases[26, 28, 29]. While existing studies have focused on a single type of RNA modification, the correlations of multiple RNA modifications in RA are not fully investigated. There were studies reported that RNA modifications played an indispensable role in inflammation, innate immunity through interaction with various modification-molecules[30]. Thus, established the overall understanding of cross-talk among these modifications was significant and meaningful. In this study, we revealed overall change of m6A, m5C, m1 A, m7G, A-to-I, $\Psi$ modification-related enzymes at transcriptional levels and mutual relationships in RA. As shown, METTL3, ALKBH5, TET2, ALYREF, TRMT10C, TRMT61B, ALKBH3, METTL 1 and ADAR1 had different mRNA expression levels in peripheral blood between RA patients and HD, which indicated these modifications might produce effects in the disease course of RA.

The level of METTL1, one of the main members of the "writers" of m7G, was decreased in RA patients. It had reported that METTL1 could constructed $\mathrm{m} 7 \mathrm{G}$ modification to regulated let-7a expression[31], while let-7a could regulated the level of Anti-CCP and was decreased in macrophages of RA [32]. These evidences hinted METT1 might play an important role in RA. In our results, METTL 1 was most related with the m6A main "writer" METTL3. This result indicated that an underlying link between these two modifications might be present. 
The modifications that occurred on adenine contained m6A, m1A, A-to-l in our study. Among these modifications, m6A was the most abundant and the most studied RNA edition. METTL3 was the key element of the m6A methyltransferases complex while ALKBH5 was the "erasers" that specific removed m6A modification. Increased METTL3 and decreased ALKBH5 in the peripheral blood of RA patients were showed in our results. Meanwhile, we also found the $\mathrm{m} 6 \mathrm{~A}$ level was increased in the RA patient (data was not show). These findings were in line with results from other published studies increased the credibility of our work[33, 34]. Besides, METTL3 was correlated with RF and DAS28-ESR and $A L K B H 5$ was related with ESR which suggested that RNA m6A modification might associate with autoantibodies and inflammation in RA. As for A-to-I modification,there were reports demonstrated ADAR1 through A-to-I RNA editing to balance immune activation and self-tolerance[35]. Other researchers had found increased expression of ADAR1 and edition level of A-to-l in the peripheral blood of RA[36], which was consistent with our work. These evidences implied ADAR1 had tightly correlation with the pathogenesis of RA. Previous study showed that there was a mainly negative correlation and a competitive relationship between m6A and A-to-I RNA editing[37]. But we could not draw a similar conclusion from our results (increased levels of METTL3 and ADAR1). The correlation analysis also showed there was no strong relation between these two molecules. But recently, a study found that m6A modification of ADAR1 mRNA results in upregulation of ADAR1 expression. Maybe the same mechanism was also existed in RA. Further research was needed for elucidated the mechanism of this phenomenon

Apart from N6 methylation and deamination, adenosines in mRNAs can also be methylated at the N1 position. The complex TRMT61BTRMT10C mainly catalyzed m1A in mitochondrial mRNA and played a vitrail role in maintaining mitochondrial basic function and metabolism through regulated the level of m1A. It was recognized mitochondrion was close related with inflammation[38-40]. The levels of both two genes were decreased in RA patients which indicated the m1A level in mitochondrial mRNA might be lower in RA patients. Yet the level of "eraser" of m1A, $A L K B H 3$, was decreased. These results raised the difficulties of explore m1A level in RA. Thus, the real aspect of m1A in RA patients need further studies to investigate. TRMT10C had a direct relationship with ESR and DAS28-ESR level. Simultaneously, the mRNA level of TRMT10C had statistically significant difference in the different subgroup of ESR and DAS28-ESR. In conclusion, it was possible that altered expression of TRMT10C caused the development of inflammation in RA. Meanwhile, $A L K B H 3$ expression level was related with ESR. All of these results indicated RNA m1A modification might play a role of inflammation in RA. As a part of the complex, TRMT61B was moderate related with WTAP. Besides that, FTO, the "eraser" of m6A modification, had strong positive correlations with m1A molecule TRMT6. It has been reported that FTO could remove m1A in tRNA, which also installed by TRMT6[41]. These results clued the possibility that FTO could remove m1A in mRNA. But until now, the study that insight into the crosstalk of m6A and m1A level did not exist. Further studies will be needed to understand this crosstalk.

The level of $A L Y R E F$ was lower while the level of TET2 was higher in RA patients. TET2, usually known as a DNA modification enzyme, also could encoded the protein catalyzes the $\mathrm{m} 5 \mathrm{C}$ to $\mathrm{hm} 5 \mathrm{c}$ in RNA[42]. Recent researches demonstrated TET2 mediates oxidation of m5C in SOCS3 mRNA to decrease SOCS3 expression level[43]. But some researchers found TET2 repress the activation of B cell through mediated chromatin modification[44]. Decrease TET2 could increase IL-1 $\beta$ level and develop inflammation[45]. Besides, other research revealed that increased TET2 play a vital part in autoimmune disease[46]. ALYREF, shown in the result, was a protective factor in RA. In previous studies, ALYREF could participate in a variety of mRNA export by combining m5C modification in mRNA[47]. Our results indicated that ALYREF had a strong positive correlation with the main m6A writer WTAP. This finding implied that ALYREF could play a potential role in m6A modification. A report proved that ALYREF could act as a genuine interaction partner of the m6A demethylase ALKBH5 and directly interacts with ALKBH5 to induce m6A removal[48]. The decreased level of $A L Y R E F$ and $A L K B H 5$ also could demonstrate the higher level of m6A modification in RA patients from the side. Meanwhile, increased TET2 and decreased ALYREF implyied m5C is the key player in pathogenesis of RA. Besides, these two genes were the most valuable risk or protective factors respectively among those molecules in RA by statistical analysis. They played high-performance in distinguished RA patients. Furthermore, no correlation was observed between these two and clinical indicators suggested both of them might be significant for the patients with negative serological.

Though the levels of $\Psi$ modification enzymes in RA patients were not differing from healthy controls, several correlations were existed between them and the molecules of $\mathrm{m} 1 \mathrm{~A}, \mathrm{~m} 5 \mathrm{C}$ modifications. A report showed that simultaneous modification by $\psi$ and m5C greatly enhanced reporter gene expression in mice and multiple cell lines. Furthermore, compared to m5C or $\Psi$ mRNA, mRNA containing both m1-pseudouridine and m5C showed a higher translational rate in vivo[49]. These findings demonstrated that $\psi$ edition was in complex interactions with other RNA modifications. However, the mechanism of the combinatorial effects of the different modifications in RA was unclear. It would be an interesting question to tackle in the future.

The routine examination of blood was the most basic and indispensable laboratory inspection. The situation of peripheral blood could assist clinic to estimate the activity of RA[50,51]. In the analysis of the correlation with blood routine, the decreased $A L K B H 5$ level in RA was negatived with PLT, while METTL3 level was increased in RA patients and positively correlated with PDW. Previous studies found the increased number of platelets could reflect the diseased activity of RA[52]. Besides, it was reported that PDW was increased in RA patients and had positive correlation with ESR and CRP[53]. The decreased levels of ALYREF and TRMT61B in RA patients were both negatived with MCHC. The 
level of MCHC was reported increased in RA. Meanwhile, it was related with inflammation markers[51]. Thus, we speculated these four genes level may indicate same clinical significance with PLT, PDW or MCHC in estimating the activity of RA.

\section{Conclusions}

Our study focused on the modifications, which were common in mRNA, and constructed a global expression network of six kinds of RNA modifications. Results demonstrated the complex relationships between these molecules and clinical parameters of RA. Furthermore, changed levels of TET2 and ALYREF in peripheral blood cell were the most valuable protective or risk factors for RA. This work helped developing a comprehend of RA pathogenic mechanism from new insight.

\section{Abbreviations}

RA: rheumatoid arthritis; m6A: N6-methyladenosine; m1A: N1-methyladenosine; m5C: 5-methylcytosine; m7G: N7-methylguanosine; $\Psi$ : pseudouridine; A-to-I: adenosine-to-inosine; RT-PCR: real time-polymerase chain reaction; LASSO: least absolute shrinkage and selection operator regression; CDS: coding for amino acids in protein; DAS28-ESR: The 28-joint disease activity score based on ESR; EULAR: European League Against Rheumatism; VAS-GH: visual analog scale; METTL3: Methyltransferase 3, N6-Adenosine-methyltransferase complex catalytic

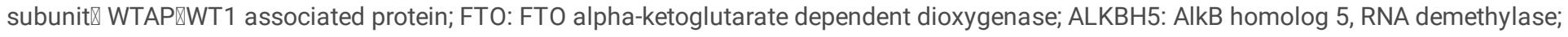
ALKBH3: $\quad$ AlkB homolog 3, alpha-ketoglutarate dependent dioxygenase; ALKBH3: $\quad$ AlkB homolog 1, histone H2A dioxygenase; TRMT10C: $\quad$ TRNA methyltransferase 10C, mitochondrial RNase P subunit; TRMT6: TRNA methyltransferase 6 non-catalytic subunit; TRMT61A: TRNA methyltransferase 61A; TRMT61B: TRNA methyltransferase 61B; ADAR1: adenosine deaminase RNA specific; ADAR2: adenosine deaminase RNA Specific B1; ALYREF: Aly/REF export factor; DNMT: DNA methyltransferase-2; NSUN2: NOP2/Sun RNA methyltransferase 2; TET2: $\quad$ Tet methylcytosine dioxygenase 2; YBX1: Y-Box binding protein 1; METTL1: methyltransferase 1, TRNA methylguanosine; RNMT: $\quad$ RNA Guanine-7 methyltransferase; WDR4: WD repeat domain 4; PUS1: pseudouridine synthase 1; PUS10: pseudouridine synthase 1; PUS7: $\quad$ pseudouridine synthase 7; TRUB1: TruB pseudouridine synthase family member 1; TRUB2: TruB pseudouridine synthase family member 2; CRP: C-reactive protein; ESR: erythrocyte sedimentation rate; Anti-CCP: anti-cyclic citrullinated peptide antibody; RF: rheumatoid factor. DAS28-ESR; PLT: platelet; HCT: red blood cell specific volume; HDW: hemoglobin distribution width; HGB: hemoglobin; MCV: mean corpusular volume; $\mathrm{MCH}$ : the level of mean corpsular hemoglobin; MCHC: mean corpuscular hemoglobin concentration; RDW: red blood cell distribution width; PCT: platelet hematocrit; MPC: mean platelet component concentration; MPM: mean platelet mass; MPV: mean platelet volume; PDW: platelet distribution width.

\section{Declarations}

\section{Acknowledgment}

We thanked the supports provided by Department of Laboratory Medicine of the First Affiliated Hospital of Fujian Medical University, Fujian Key Laboratory of Laboratory Medicine and Gene Diagnosis Research Center, Fujian Medical University.

\section{Authors' contributions}

Conceptualization: JL, JT and ZY. Methodology: major contribution by YX, JX, SC and RJ. Statistics analysis: contribution by JT and ZY. Writing original draft: ZY. Review and editing: contribution by JL, YH and JT. Funding acquisition, project administration: JL. All authors contributed to the article and approved the submitted version.

\section{Funding}

This work was supported by grant from National Natural Science Foundation of China, China (82072356), Program for young and middle-aged backbone of Fujian Provincial Health Commission, China (2019-ZQN-53). and Fujian Medical University Startup Fund for scientific research, China (2018QH1056).

\section{Availability of data and materials}

The raw data supporting the conclusions of this article will be made available by the authors, without undue reservation.

\section{Ethics approval and consent to participate}

The studies involving human participants were reviewed and approved by Ethics Committee of the First Affiliated Hospital of Fujian Medical University Document NO. IEC-FOM-013-2.0. The participants provided their written informed consent to participate in this study. 
Consent for publication

Not applicable.

\section{CONFLICT OF INTEREST}

The authors declare no conflict of interest.

Competing interests

The authors declare no competing interests.

\section{References}

1. Weyand CM, Goronzy JJ: The immunology of rheumatoid arthritis. Nat Immuno/2020.

2. Boccaletto P, Machnicka MA, Purta E, Piatkowski P, Baginski B, Wirecki TK, de Crécy-Lagard V, Ross R, Limbach PA, Kotter Aet al: MODOMICS: a database of RNA modification pathways. 2017 update. Nucleic Acids Res2018, 46(D1):D303-d307.

3. Shi H, Chai P, Jia R, Fan X: Novel insight into the regulatory roles of diverse RNA modifications: Re-defining the bridge between transcription and translation. Mol Cancer2020, 19(1):78.

4. Wei CM, Gershowitz A, Moss B: Methylated nucleotides block 5' terminus of HeLa cell messenger RNA. Cel/1975, 4(4):379-386.

5. Cohn WE: Pseudouridine, a carbon-carbon linked ribonucleoside in ribonucleic acids: isolation, structure, and chemical characteristics. The Journal of biological chemistry1960, 235:1488-1498.

6. Dubin DT, Taylor RH: The methylation state of poly A-containing messenger RNA from cultured hamster cells. Nucleic Acids Res1975, 2(10):1653-1668.

7. Kietrys AM, Kool ET: Epigenetics: A new methyl mark on messengers. Nature2016, 530(7591):423-424.

8. Wiener D, Schwartz S: The epitranscriptome beyond m(6)A. Nat Rev Genet2020.

9. Zheng HX, Zhang XS, Sui N: Advances in the profiling of N(6)-methyladenosine (m(6)A) modifications. Biotechnol AdV2020, 45:107656.

10. Li X, Xiong X, Zhang M, Wang K, Chen Y, Zhou J, Mao Y, Lv J, Yi D, Chen XWet al: Base-Resolution Mapping Reveals Distinct m(1)A Methylome in Nuclear- and Mitochondrial-Encoded Transcripts. Mol Cel/2017, 68(5):993-1005 e1009.

11. Safra M, Sas-Chen A, Nir R, Winkler R, Nachshon A, Bar-Yaacov D, Erlacher M, Rossmanith W, Stern-Ginossar N, Schwartz S: The m1A landscape on cytosolic and mitochondrial mRNA at single-base resolution. Nature2017, 551(7679):251-255.

12. Dominissini D, Nachtergaele S, Moshitch-Moshkovitz S, Peer E, Kol N, Ben-Haim MS, Dai Q, Di Segni A, Salmon-Divon M, Clark WCet al: The dynamic N(1)-methyladenosine methylome in eukaryotic messenger RNA. Nature2016, 530(7591):441-446.

13. Pestal K, Funk CC, Snyder JM, Price ND, Treuting PM, Stetson DB: Isoforms of RNA-Editing Enzyme ADAR1 Independently Control Nucleic Acid Sensor MDA5-Driven Autoimmunity and Multi-organ Development. Immunity2015, 43(5):933-944.

14. Eisenberg E, Levanon EY: A-to-I RNA editing - immune protector and transcriptome diversifier. Nat Rev Genet2018, 19(8):473-490.

15. Carlile TM, Rojas-Duran MF, Zinshteyn B, Shin H, Bartoli KM, Gilbert WV: Pseudouridine profiling reveals regulated mRNA pseudouridylation in yeast and human cells. Nature2014, 515(7525):143-146.

16. Schwartz S, Bernstein DA, Mumbach MR, Jovanovic M, Herbst RH, Leon-Ricardo BX, Engreitz JM, Guttman M, Satija R, Lander ES et al: Transcriptome-wide mapping reveals widespread dynamic-regulated pseudouridylation of ncRNA and mRNA. Cel/2014, 159(1):148-162.

17. Mukhopadhyay S, Deogharia M, Gupta R: Mammalian nuclear TRUB1, mitochondrial TRUB2, and cytoplasmic PUS10 produce conserved pseudouridine 55 in different sets of tRNA. RNA (New York, NY)2021, 27(1):66-79.

18. Ramanathan A, Robb GB, Chan SH: mRNA capping: biological functions and applications. Nucleic Acids Res2016, 44(16):7511-7526. 
19. Zhao Y, Kong L, Pei Z, Li F, Li C, Sun X, Shi B, Ge J: m7G Methyltransferase METTL1 Promotes Post-ischemic Angiogenesis via Promoting VEGFA mRNA Translation. Front Cell Dev Bio/2021, 9:642080.

20. Varshney D, Lombardi O, Schweikert G, Dunn S, Suska O, Cowling VH: mRNA Cap Methyltransferase, RNMT-RAM, Promotes RNA Pol IIDependent Transcription. Cell Rep2018, 23(5):1530-1542.

21. Bohnsack KE, Hobartner C, Bohnsack MT: Eukaryotic 5-methylcytosine (m(5)C) RNA Methyltransferases: Mechanisms, Cellular Functions, and Links to Disease. Genes (Basel)2019, 10(2).

22. Wang H, Hu X, Huang M, Liu J, Gu Y, Ma L, Zhou Q, Cao X: Mett|3-mediated mRNA m(6)A methylation promotes dendritic cell activation. Nat Commun2019, 10(1):1898.

23. Du J, Liao W, Liu W, Deb DK, He L, Hsu PJ, Nguyen T, Zhang L, Bissonnette M, He Cet al: N(6)-Adenosine Methylation of Socs1 mRNA Is Required to Sustain the Negative Feedback Control of Macrophage Activation. Dev Cel/2020.

24. HB L, J T, S Z, PJ B, EE D, J Z, W B, G C, L K, Y Cet al: mA mRNA methylation controls T cell homeostasis by targeting the IL-7/STAT5/SOCS pathways. Nature2017, 548(7667):338-342.

25. Wang N, Tang H, Wang X, Wang W, Feng J: Homocysteine upregulates interleukin-17A expression via NSun2-mediated RNA methylation in T lymphocytes. Biochem Biophys Res Commun2017, 493(1):94-99.

26. Guo G, Wang H, Shi X, Ye L, Yan K, Chen Z, Zhang H, Jin Z, Xue X: Disease Activity-Associated Alteration of mRNA m(5) C Methylation in CD4(+) T Cells of Systemic Lupus Erythematosus. Front Cell Dev Bio/2020, 8:430.

27. Devarkar SC, Wang C, Miller MT, Ramanathan A, Jiang F, Khan AG, Patel SS, Marcotrigiano J: Structural basis for m7G recognition and 2'0-methyl discrimination in capped RNAs by the innate immune receptor RIG-I. Proc Natl Acad Sci U S A2016, 113(3):596-601.

28. Li LJ, Fan YG, Leng RX, Pan HF, Ye DQ: Potential link between m(6)A modification and systemic lupus erythematosus. Mol Immuno/2018, 93:55-63.

29. Luo Q, Fu B, Zhang L, Guo Y, Huang Z, Li J: Decreased Peripheral Blood ALKBH5 Correlates with Markers of Autoimmune Response in Systemic Lupus Erythematosus. Dis Markers2020, 2020:8193895.

30. Chen H, Yao J, Bao R, Dong Y, Zhang T, Du Y, Wang G, Ni D, Xun Z, Niu Xet al: Cross-talk of four types of RNA modification writers defines tumor microenvironment and pharmacogenomic landscape in colorectal cancer. Mol Cancer2021, 20(1):29.

31. Pandolfini L, Barbieri I, Bannister AJ, Hendrick A, Andrews B, Webster N, Murat P, Mach P, Brandi R, Robson SCet al: METTL1 Promotes let-7 MicroRNA Processing via m7G Methylation. Mol Cel/2019, 74(6):1278-1290 e1279.

32. Zhu W, Yu J, Qiu S, Liu H, Wang Y, Xu X, Shao L, Zhu L, Jiao Y, Liu Fet al: MiR-let-7a regulates anti-citrullinated protein antibody-induced macrophage activation and correlates with the development of experimental rheumatoid arthritis. Int Immunopharmaco/2017, 51:40-46.

33. Luo Q, Gao Y, Zhang L, Rao J, Guo Y, Huang Z, Li J: Decreased ALKBH5, FTO, and YTHDF2 in Peripheral Blood Are as Risk Factors for Rheumatoid Arthritis. Biomed Res Int2020, 2020:5735279.

34. Wang J, Yan S, Lu H, Wang S, Xu D: METTL3 Attenuates LPS-Induced Inflammatory Response in Macrophages via NF-kappaB Signaling Pathway. Mediators Inflamm2019, 2019:3120391.

35. Lamers MM, van den Hoogen BG, Haagmans BL: ADAR1: "Editor-in-Chief" of Cytoplasmic Innate Immunity. Front Immuno/2019, 10:1763.

36. Vlachogiannis NI, Gatsiou A, Silvestris DA, Stamatelopoulos K, Tektonidou MG, Gallo A, Sfikakis PP, Stellos K: Increased adenosine-toinosine RNA editing in rheumatoid arthritis. J Autoimmun2020, 106:102329.

37. Xiang JF, Yang Q, Liu CX, Wu M, Chen LL, Yang L: N(6)-Methyladenosines Modulate A-to-I RNA Editing. Mol Cel/2018, 69(1):126-135 e126.

38. Vizioli MG, Liu T, Miller KN, Robertson NA, Gilroy K, Lagnado AB, Perez-Garcia A, Kiourtis C, Dasgupta N, Lei Xet al: Mitochondria-to-nucleus retrograde signaling drives formation of cytoplasmic chromatin and inflammation in senescence. Genes Dev2020, 34(5-6):428-445.

39. Green DR, Galluzzi L, Kroemer G: Mitochondria and the autophagy-inflammation-cell death axis in organismal aging. Science2011, 333(6046):1109-1112. 
40. Gabande-Rodriguez E, Gomez de Las Heras MM, Mittelbrunn M: Control of Inflammation by Calorie Restriction Mimetics: On the Crossroad of Autophagy and Mitochondria. Cells2019, 9(1).

41. Wei J, Liu F, Lu Z, Fei Q, Ai Y, He PC, Shi H, Cui X, Su R, Klungland Aet al: Differential m(6)A, m(6)Am, and m(1)A Demethylation Mediated by FTO in the Cell Nucleus and Cytoplasm. Mol Cel/2018, 71(6):973-985 e975.

42. He C, Bozler J, Janssen KA, Wilusz JE, Garcia BA, Schorn AJ, Bonasio R: TET2 chemically modifies tRNAs and regulates tRNA fragment levels. Nat Struct Mol Bio/2021, 28(1):62-70.

43. Shen Q, Zhang Q, Shi Y, Shi Q, Jiang Y, Gu Y, Li Z, Li X, Zhao K, Wang Cet al: Tet2 promotes pathogen infection-induced myelopoiesis through mRNA oxidation. Nature2018, 554(7690):123-127.

44. Tanaka S, Ise W, Inoue T, Ito A, Ono C, Shima Y, Sakakibara S, Nakayama M, Fujii K, Miura let al: Tet2 and Tet3 in B cells are required to repress CD86 and prevent autoimmunity. Nat Immuno/2020, 21(8):950-961.

45. Potus F, Pauciulo MW, Cook EK, Zhu N, Hsieh A, Welch CL, Shen Y, Tian L, Lima P, Mewburn Jet al: Novel Mutations and Decreased Expression of the Epigenetic Regulator TET2 in Pulmonary Arterial Hypertension. Circulation2020, 141(24):1986-2000.

46. Lagos C, Carvajal P, Castro I, Jara D, González S, Aguilera S, Barrera MJ, Quest AFG, Bahamondes V, Molina Cet al: Association of high 5hydroxymethylcytosine levels with Ten Eleven Translocation 2 overexpression and inflammation in Sjögren's syndrome patients. Clinical immunology (Orlando, Fla)2018, 196:85-96.

47. Fan J, Wang K, Du X, Wang J, Chen S, Wang Y, Shi M, Zhang L, Wu X, Zheng Det al: ALYREF links 3'end processing to nuclear export of non-polyadenylated mRNAs. EMBO J2019, 38(9).

48. Zheng G, Dahl JA, Niu Y, Fedorcsak P, Huang CM, Li CJ, Vågbø CB, Shi Y, Wang WL, Song SHet al: ALKBH5 is a mammalian RNA demethylase that impacts RNA metabolism and mouse fertility. Mol Cel/2013, 49(1):18-29.

49. Andries O, Mc Cafferty S, De Smedt SC, Weiss R, Sanders NN, Kitada T: N(1)-methylpseudouridine-incorporated mRNA outperforms pseudouridine-incorporated mRNA by providing enhanced protein expression and reduced immunogenicity in mammalian cell lines and mice. J Control Release2015, 217:337-344.

50. Yazici S, Yazici M, Erer B, Erer B, Calik Y, Ozhan H, Ataoglu S: The platelet indices in patients with rheumatoid arthritis: mean platelet volume reflects disease activity. Platelets2010, 21(2):122-125.

51. Moller B, Everts-Graber J, Florentinus S, Li Y, Kupper H, Finckh A: Low Hemoglobin and Radiographic Damage Progression in Early Rheumatoid Arthritis: Secondary Analysis From a Phase III Trial. Arthritis Care Res (Hoboken)2018, 70(6):861-868.

52. Zhou Z, Chen H, Ju H, Sun M, Jin H: Platelet indices in patients with chronic inflammatory arthritis: a systematic review and meta-analysis. Platelets2020, 31(7):834-844.

53. Khaled SAA, NasrEldin E, Makarem YS, Mahmoud HFF: Value of Platelet Distribution Width and Mean Platelet Volume in Disease Activity Score of Rheumatoid Arthritis. J Inflamm Res2020, 13:595-606.

\section{Tables}

Due to technical limitations, Table 3 and 5 is only available as a download in the Supplemental Files section.

\section{Figures}



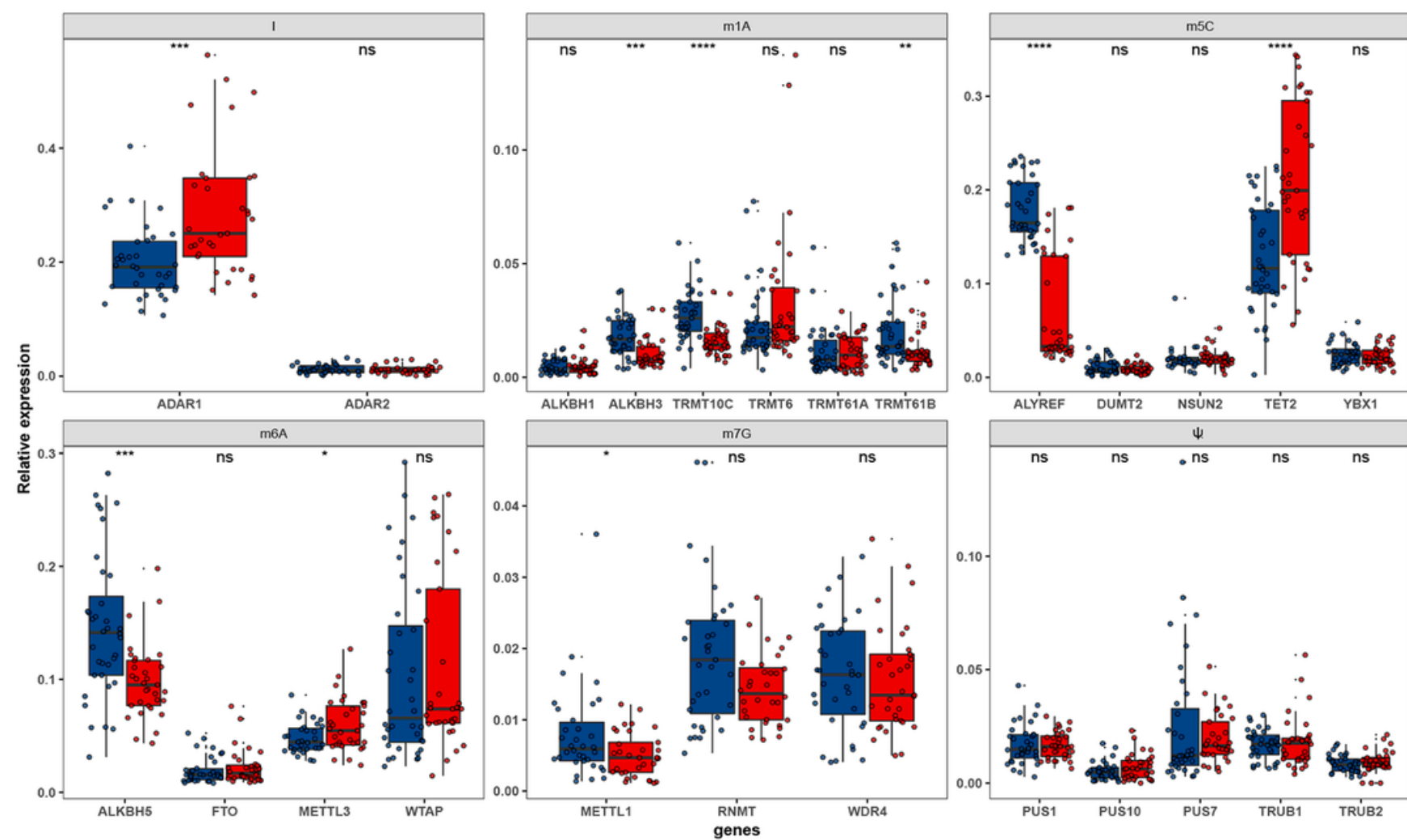

Groups

\section{Figure 1}

Box-plot diagram of the mRNA expression of six kinds of RNA modification-related molecules between RA (red) and HD (blue). The median, interquartile range, and outliers were shown in the graph. Plot was created by ggplot2 package in $\mathrm{R}$ software. ${ }^{\star} \mathrm{p}<0.05,{ }^{\star \star} \mathrm{p}<0.01,{ }^{\star \star \star} \mathrm{p}<0.001$, $\star \star \star \star ~ p<0.0001$. 


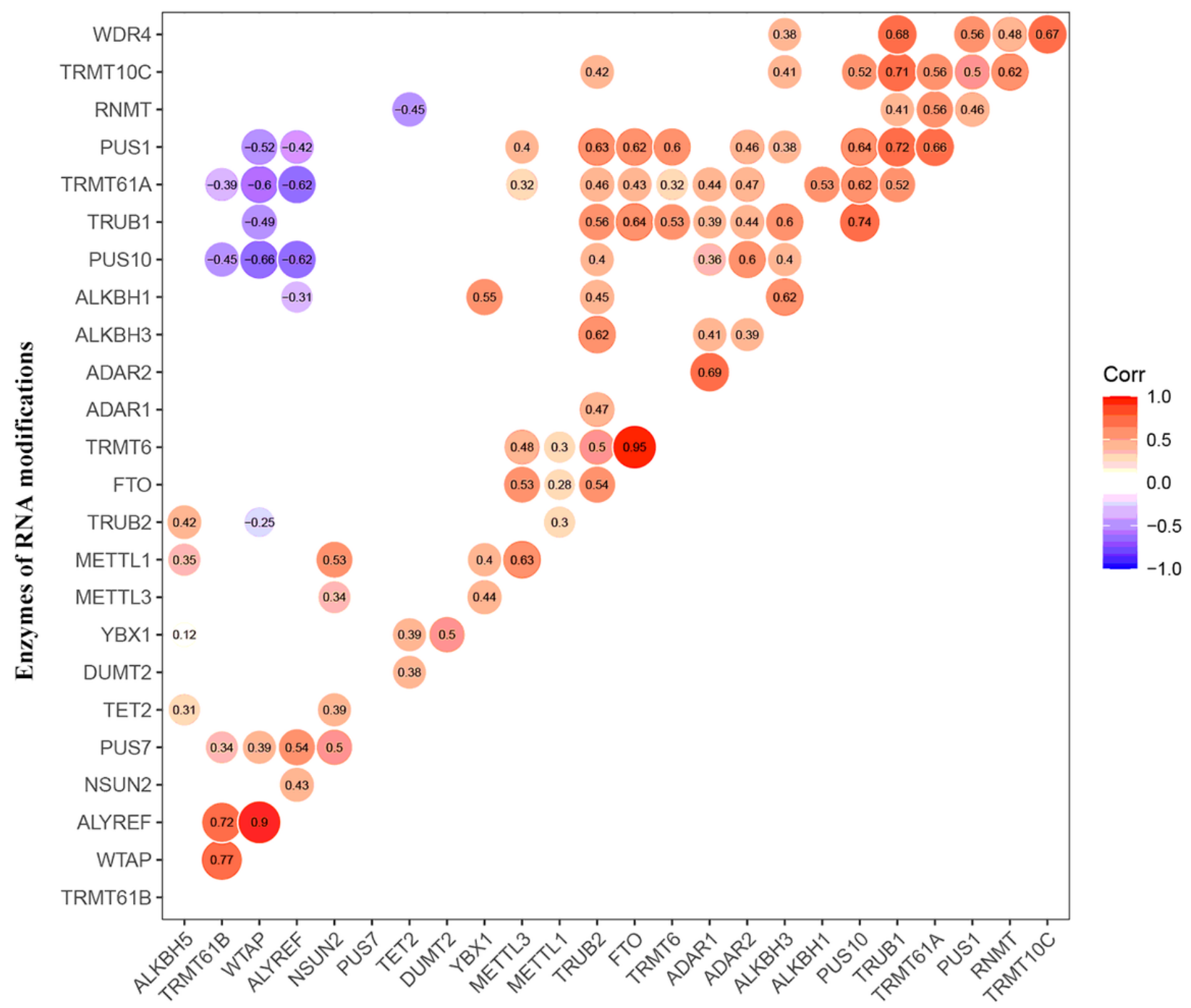

Enzymes of RNA modifications

\section{Figure 2}

Heatmap of the correlation among six kind of RNA modification-related molecules in RA patients. When p<0.05 in Person correlation analysis, the spot was visible. Positive or negative correlation was shown in red or blue. The size of circle represents $r$ value. Plot was created by ggcorrplot package in R software. 
A

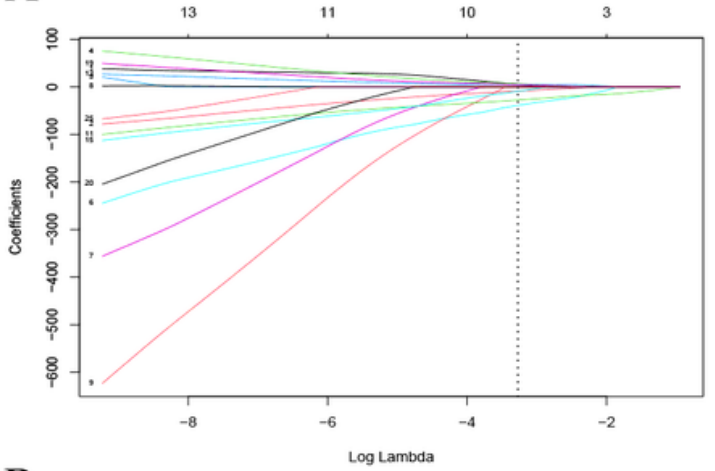

$\mathrm{B}$

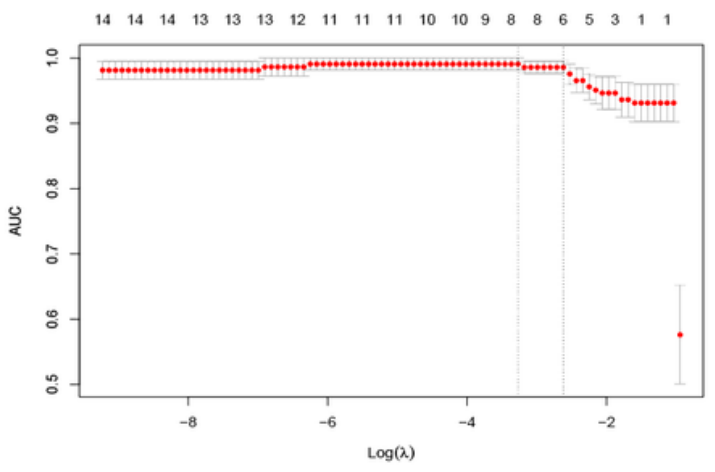

$\mathrm{C}$

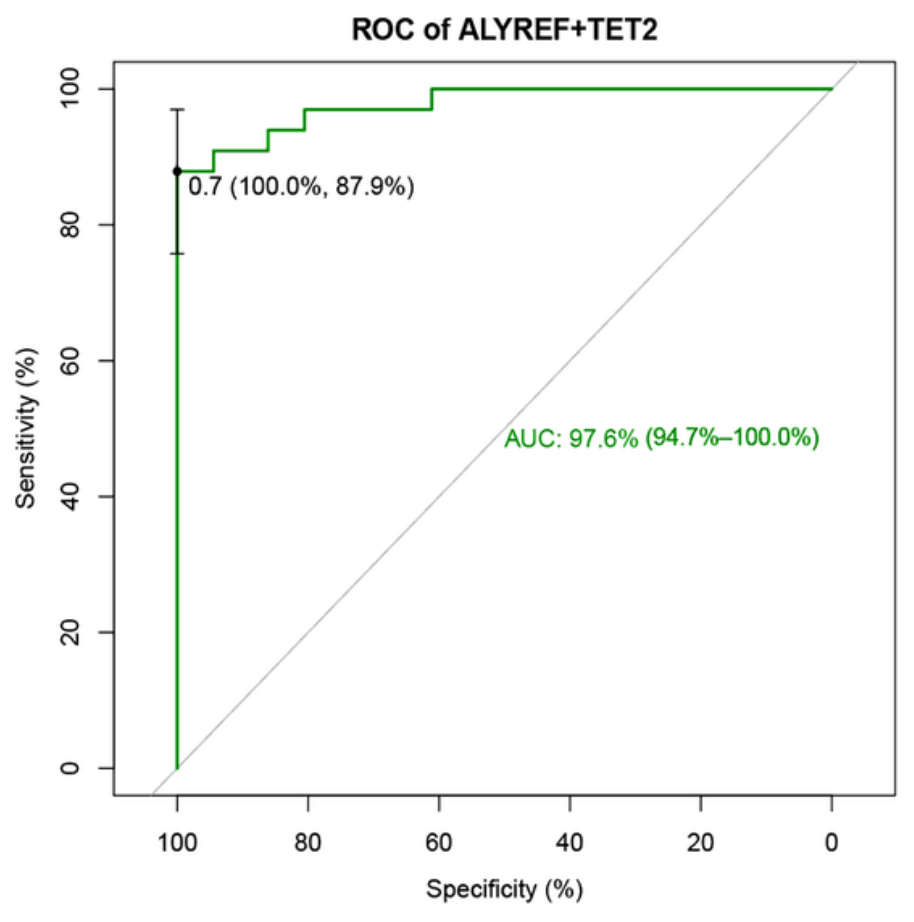

\section{Figure 3}

Exploring the possible biomarkers for distinguished RA patients and healthy controls. (A) LASSO coefficient profiles of the 27 features. A coefficient profile plot was produced against the $\log \lambda$ sequence. Vertical line was drawn at the value selected using 5 -fold cross-validation, where optimal lambda resulted in 8 variables with non-zero coefficients. (B) The relationship curve between AUC and $\log \lambda$ was plotted. A vertical line was drawn at the value chosen by five-fold cross-validation, which indicated that 8 non-zero coefficients were identified by the optimal lambda value, Min $\lambda=0.03791$. The dashed line on the right is a more concise model within one standard error (SE), SE=0.009310. (C) ROC analysis was performed to evaluate the performance of the mRNA expression levels of ALYREF combined with TET2 in distinguishing RA from $\mathrm{HD}, \mathrm{AUC}=0.976>0.8$.

\section{Supplementary Files}

This is a list of supplementary files associated with this preprint. Click to download.

- Table3and5.docx

- sup.xlsx 